Article

\title{
INVESTIGATION INTO RESTORATION EXPERIENCE: THE EFFECTS OF PERCEIVED SENSORY DIMENSION AND PERCEIVED RESTORATIVENESS
}

\author{
Fahimeh Malekinezhad ${ }^{1, *}$ and Hasanuddin Bin Lamit ${ }^{2}$ \\ 1 Faculty of Built Environment, Universiti Teknologi Malaysia; mfahimeh2@utm.my \\ 2 Faculty of Built Environment, Universiti Teknologi Malaysia; b-hasanuddin@utm.my \\ * Correspondence: mfahimeh2@utm.my
}

\begin{abstract}
Salutogenic landscape with its health-promoting qualities is a solution for ever-increasing problem of stress for university students as a vulnerable group. Based on nature related theories, natural environments could have a salutary effect on psychological restoration through stress alleviation. There is a body of research on investigating the impact of open green space characteristics for increasing of psychological restoration through framework of attention restoration theory and supportive environment theory. However, there is little knowledge on how the interaction among these characteristics could promote perceived restoration experience (PRE). To address this problem, using perceived sensory dimension (PSD) for open green space qualities and Perceived Restorativeness (PR) as properties of restorative environments, this study assessed the extent to which the PSD and PR impact on PRE. Using 444 screened data, the mediation effect of PR on the association of PSD to PRE is shown as well as the validity and reliability of proposed relationships. 'Serene', 'space', 'prospect', 'rich in species' and 'nature' were the most influential PSDs on PRE through their higher effect on perceived characteristics of 'fascination' and 'compatibility'. These results provide information within landscape architecture and planning to promote the development of open spaces as resources for psychological restoration and stress relief.
\end{abstract}

Keywords: perceived sensory dimension (PSD); restoration experience; perceived restorativeness; university campus open space; university students

\section{Introduction}

Stress and mental fatigue are the greatest risk factor of psychological well-being and mental health in human life (Asztalos et al. 2009), specifically among university students (Baghurst and Kelley 2014). Restoration experience in green spaces is suggested as a positive action against excess stress. It is recharging of the psychological, physiological and emotional capacities, which are diminished in performing of daily life tasks (Han 2003). Perceived restorativeness characteristics have acted as the supportive mechanism on the effect of environment qualities and positive outcomes (Hipp et al. 2016; Marselle et al. 2016). Salutogenic landscapes with a set of specific characteristics are suggested for mental restoration as some were shown to be more influential than others in this process (Grahn and Stigsdotter 2010). Still, it is not clear how the addressed salutary factors of green spaces are related with perceived restorativeness characteristics and restoration experience. Although, understanding the significance of underlying measurement variables urged by many studies (e.g. Hipp et al. 2016; Marselle et al. 2016), there are few to take the approach for understanding the 
university students psychological restoration through salutary factors and perceived restorativeness characteristics that presented in campus green spaces. Therefore, this study integrates nature related theories to explain how and why restoration experience is happening and what are the important qualities for experience of psychological restoration, as it could provide valuable information to design psychological health-promoting environments in general and specifically for university students.

\subsection{Perceived Restoration Experience (PRE)}

The association between human interaction with nature has been investigated through the actual and perceived restoration experiences. The actual restoration experience studies through laboratory or field experimental design approaches monitored the beneficial changes of short exposure to nature on subjects' psycho-physiological and emotional state (e.g. Nordh et al. 2009). The perceived restoration experience studies through a cross-sectional or longitudinal design approach found the beneficial effects of repeated exposure to nature in subjects everyday settings (e.g. Hansmann et al. 2007). Referring to the Biophilia hypothesis, stress recovery theory (SRT) and attention restoration theory (ART), the concurrence is on restoration experience through both direct and indirect interaction with nature. The Biophilia hypothesis explains human evolutionary affiliation to nature and such connectedness to nature shapes experience of positive outcomes (e.g. Haluza et al. 2014). From the viewpoint of SRT, natural environments rapidly evoke affective responses and reduce high stress (Bratman et al. 2015). The ART studies asserted that natural environments with soft fascinating objects can provide opportunities for psychological restoration as does not require conscious mind efforts (e.g. Kaplan 1995; Pals et al. 2009).

\subsection{Perceived Restorativeness (PR)}

The concept of Perceived restorativeness is developed in ART. Perceived restorativeness is insight into perceptions toward restorativeness qualities of physical environments (Ivarsson and \& Hagerhall 2008; Scopelliti et al. 2012). There are four components in perceived restorativeness as 'being away', 'fascination', 'extent' and 'compatibility' (Kaplan 1995). Perceive quality of 'being away' is when one's feel is psychologically and physically away from everyday environment and demands. Perceiving quality of 'fascination' is when environment offers fascinating objects that attract attention effortlessly. Perceiving of 'extent' quality is when environment has sufficient objects to engage one's mind. Perceive quality of 'compatibility' is when there is a perfect match between a person and that environment (Kaplan 1995).

In conceptualization of perceived restorativeness and perceived restoration experience, it has been explained that both concepts emphasize "the recovering aspects of places, which allow people to distract, to relax, to free their minds and to distance themselves from ordinary aspects of life" Scopelliti and Vittoria Giuliani (2004, p.423). The difference is that perceived restoration experience is the state of reduced mental fatigue and perceived restorativeness is the capacity of setting, where mental fatigue is reduced (Han 2003). Therefore, research based on perceived restorativeness approach evaluates perception towards the potential restorativeness capacity of physical environment by evaluation on the proposed components (Ivarsson and \& Hagerhall 2008). Perceived restorativeness has been considered to play a mediating role in explaining how nature can induce positive outcomes in recent studies (Hipp et al. 2016; Marselle et al. 2015 2016).

\subsection{Perceived Sensory Dimension (PSD)}

Perceived Sensory Dimension (PSD) has been introduced as the new version of open space characteristics, which has been developed by Grahn and his colleges between years of 1985 to 2010 (Grahn 1991; Grahn et al. 2005; Grahn and Stigsdotter 2010). They suggested that classification of green space characteristics is apart from qualities that have been attributed to the physical environment. In the first classification version, the introduced characteristics were 'rich variety of specie', 'wilderness', 'sports oriented', 'forest', 'play inspiring', 'festive', 'peaceful' and 'square'. In the next version, it involved 
landscape characteristics of 'serene', 'wild', 'space', 'rich in species', 'culture', 'the common', 'the pleasure garden' and 'festive' (Grahn et al. 2005). The latest one, which has been so-called as PSD consisted of eight perceived qualities as 'culture', 'nature', 'prospect', 'refuge', 'rich in species', 'social', 'space' and 'serene' (Grahn and Stigsdotter 2010). The idea in development of PSD is based on multi-sensory perception of open green spaces through tactile sensation, hearing, sight and smell and supportive environment theory (SET) Grahn and Stigsdotter (2010); Memari et al. (2017); Stoltz et al. (2016).

The green space related characteristics has been used as a valid and reliable measurement approach in landscape open spaces. For example, using the geographic information system and area-aggregated self report assessment approaches, studies showed how availability of these characteristics in nearby neighbourhoods could be associated with their residents' higher psychological health benefits (Annerstedt et al. 2012; De Jong et al. 2011). Research conducted in nature-based therapy has demonstrated how the outdoor environment with these salutary features can serve as therapeutic landscapes for stress recovery and rehabilitation (Bengtsson and Grahn 2014). The association of PSDs with evaluation of perceived restorativeness have been supported in urban open spaces Peschardt and Stigsdotter (2013) and in eliciting of restorative responses in the forest environments Stigsdotter et al. (2017). The restorative potential of physical properties of forests stands has been seen through the mediated effect of PSDs (Stoltz et al. 2016). The study by (Memari et al. 2017) highlighted the positive effect of PSDs for stress restoration. In the present study, the perception of these eight qualities in campus open spaces investigated as salutary indicators of psychological restoration.

\subsection{Aims $\mathcal{E}$ hypotheses}

In accordance with the literature on the beneficial effect of nature, the aim of this study is to investigate perceived restoration through the effect of perceived sensory dimension and perceived restorativeness characteristics (Figure 1). There are three main aims and related hypotheses as followings, which are tested in the context of university campus among population of university students:

- $\quad$ The first aim is to analysis the relationship between perceived sensory dimension and perceived restorativeness and perceived restoration experience.

- H1: Perceived sensory dimension has positive impact on perceived restoration experience.

- H2: Perceived sensory dimension has positive impact on perceived restorativeness.

- H3: Perceived restorativeness has positive impact on perceived restoration experience.

- The second aim is to analysis the effect of perceived restorativeness on the association between perceived sensory dimension and perceived restoration experience.

- H4: Perceived restorativeness is the underlying mechanism in the relationship between perceived sensory dimension and perceived restoration experience (Mediation Effect).

The third aim is to determine the role of perceived sensory dimensions and perceived restorativeness characteristics in the process of developing perceived restoration experience. There are two questions to answer as follows:

- Q1: Which measurement variables explain perceived restorativeness in campus open spaces?

- Q2: How measurement variables of perceived sensory dimension are related to perceived restorativeness and restoration experience? 


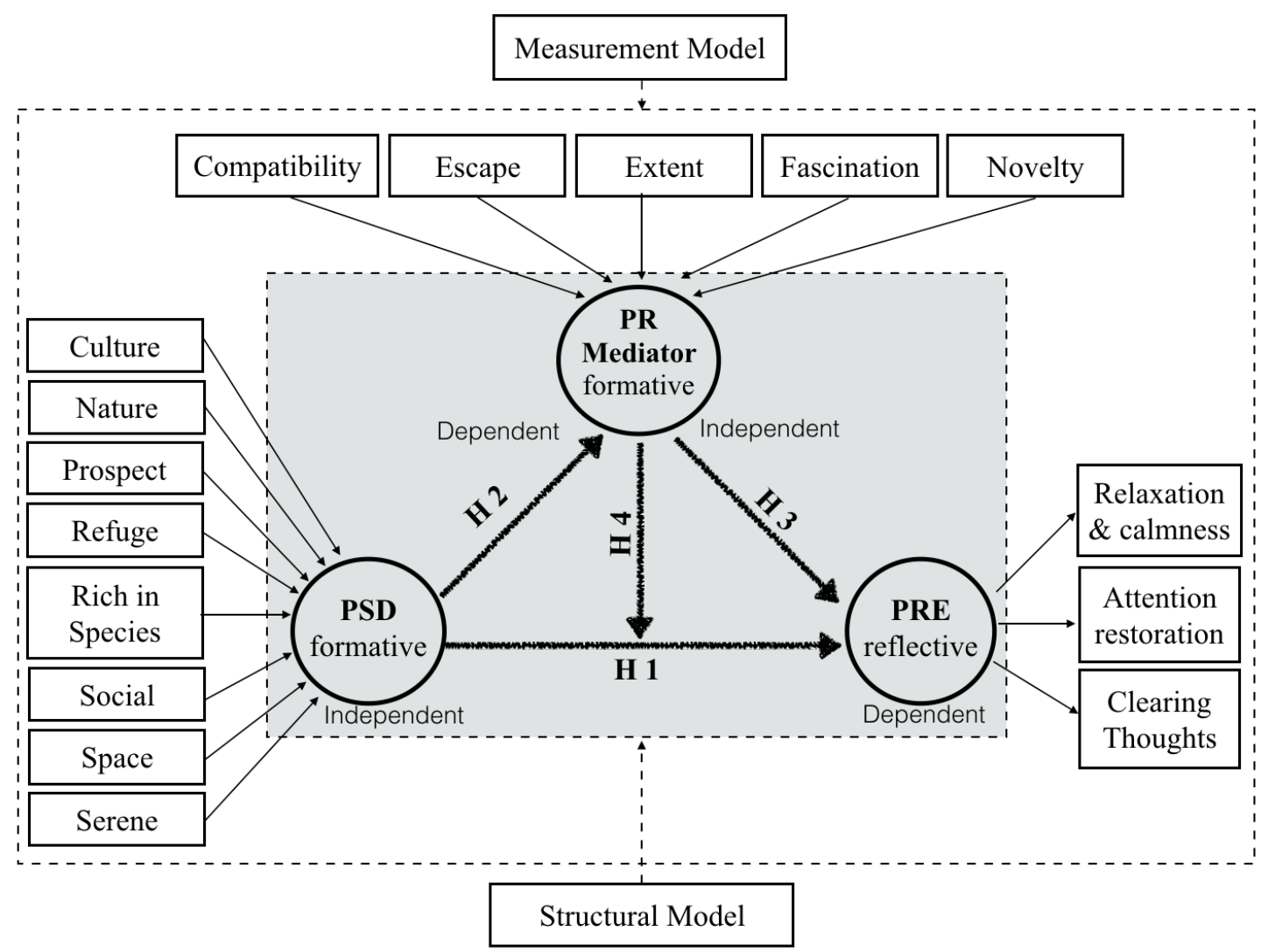

Figure 1. Path model diagram of the direct association of PSD $\rightarrow$ PRE, PSD $\rightarrow$ PR, PR $\rightarrow$ PRE (H1, H2, and H3). The measurement model contains the measurement variables of PSD and PR, which assessed as formative constructs and PRE, which assessed as reflective construct. H4 is the mediating effect of PR on association between PSD and PRE

\section{Method}

\subsection{Participants and Procedures}

A quantitative questionnaire through the collective administration approach is used for data collection. This method has been known as the quickest way of data collection to get questionnaires with a high response rate Kumar (2011). This method provides the opportunity of personal contact with respondents to explain the research purpose. This step was conducted through a sample of university students in five Malaysian Research Universities (MRU). Selection of respondents in each university was based on convenience sampling method. Following pilot testing, students were asked to rate their favourite places on university campus outdoor space, in terms of perceived green space related qualities, restorativeness characteristics and after visit feelings of restorative outcomes, which all were presented in the questionnaires.

\subsection{Measures}

Perceived Restoration Experience: the Restorative Outcome Scale (ROS) was used in assessment of restoration experience, as was done by previous studies in visit to favorite places (Korpela et al. 2008). Through ROS-6 items, the measured restorative variables are 'attention restoration', 'clearing thoughts' and 'relaxation $\&$ calmness'. In previous studies, the reliability of respondents' recalled feelings of restoration in visit to natural and urban environments has been supported (White et al. 2013). The questions in this part were measured on the 7-points Likert scale of 'not at all' to 'completely'. 
Perceived Restorativeness: the Restorative Components Scale (RCS - 22 items) was used in measurement of perceived restorativeness. Using RCS overcomes the concerns attributed to the original PRS, including the psychometric construction of items (e.g. Pasini et al. 2014). It consists of five components including 'compatibility', 'fascination', 'extent', 'novelty' and 'escape'. Compared with other developed perceived restorativeness scales (e.g. Bagot 2004; Lehto 2013), RCS does not emphasis on the aspects that make it specifically for a particular environment or population. In addition to RCS, Perceived restorativeness also was asked through a single item question for construct validity and reliability tests. The questions in this part were measured on the 7-points Likert scale of 'not at all' to 'completely'.

Perceived Sensory Dimension: PSD was assessed using eight perceived qualities including 'culture', 'nature', 'prospect', 'refuge', 'rich in species', 'social', 'space' and 'serene'. The relevant studies have supported the validity of assessment of green spaces with these eight perceived sensory dimensions (Qiu and Nielsen 2015; Stigsdotter et al. 2017), across different cultural background (Memari et al. 2017; Skärbäck et al. 2015) and in context of university campus (Skarback and Grahn 2016). In this study each of eight PSD variables is measured through 3 items, which are addressed in the PSDs' studies (e.g. Grahn and Stigsdotter 2010; Peschardt and Stigsdotter 2013), based on its relevance to the context of campus open space via expert item refinement approach (Table 1). Moreover, a single item question was asked for reliability and validity tests of this construct in addition to 24 items of PSD. The questions in this part were measured on the 7-points Likert scale of 'totally disagree' to 'totally agree'.

Table 1. PSD 24 items with its 8 dimensions

\begin{tabular}{|c|c|}
\hline Dimensions & Items \\
\hline Culture & $\begin{array}{l}\text { Contains fountain, status or pond } \\
\text { Decorated with exotic plants } \\
\text { Decorated with historical elements }\end{array}$ \\
\hline Nature & $\begin{array}{l}\text { Contains rows of trees, bushes, scent of grass } \\
\text { Has naturally growing greenery } \\
\text { Feelings of being in untouched nature }\end{array}$ \\
\hline Prospect & $\begin{array}{l}\text { Plane and well-cut lawns } \\
\text { Contains plain surfaces } \\
\text { Visibility to over surroundings }\end{array}$ \\
\hline Refuge & $\begin{array}{l}\text { Safe environment with a sense of privacy } \\
\text { Enclosed with many shrubs and trees } \\
\text { Offers sitting to watch others being active }\end{array}$ \\
\hline Rich in Species & $\begin{array}{l}\text { Contains a wide range of plants } \\
\text { Contains a wide range of birds, animals and insects } \\
\text { Contains variety of specific flowers }\end{array}$ \\
\hline Social & $\begin{array}{l}\text { Contains several seats and benches } \\
\text { Contains plenty of people and movements } \\
\text { Equipped with facilities for festivity and pleasure }\end{array}$ \\
\hline Space & $\begin{array}{l}\text { Is a free and vast environment } \\
\text { Contains lots of sunny and shady areas } \\
\text { Possible to move freely }\end{array}$ \\
\hline Serene & $\begin{array}{l}\text { Not effects of traffic noise or disturbing people } \\
\text { Is a clean and well-maintained environment } \\
\text { Contains sounds of wind, birds and water }\end{array}$ \\
\hline
\end{tabular}




\section{Statistical Analysis}

Structural Equation Modelling (SEM) is the second-generation multivariate data analysis method that combines factor analysis and multiple regressions. Although there are two types of SEM which are covariance-based SEM (CB-SEM) and partial least square SEM (PLS-SEM), the use of PLS-SEM in this study was more appropriate since it is exploratory in its nature (Hair et al. 2016). in this study a model PLS-SEM model is developed using SmartPLS v3.0 (Ringle et al. 2015). The use of SEM provides more robust model testing compared to conventional regression modelling. Prior to examining the structural model for hypotheses testing, a measurement model is assessed in this study, to determine the validity and reliability of the proposed model. The recommendations by (Hair et al. 2016) was used for all the steps of data screening step and model evaluation.

The first step before examining data by SmartPLS is data quality evaluation. Using IBM SPSS Statistics v23.0, the data screening procedures have been done on all collected data. The steps, which have been taken are checking for missing data, looking for suspicious response pattern, detecting outliers and checking for data distribution. As it has been expected, by including extra $20 \%$ in the number of respondents $(n=500)$ (De Vaus 2002), 19\% of the collected data has been removed in various steps of data screening and the remaining data for evaluation in PLS-SEM is 444 respondents.

The next phase is evaluation of model consisting of reliability and validity assessment of measurement model and structural model. In the proposed PLS-SEM path model, the measurement model contains the relationship between the latent constructs and their measurement variables, which obtained by self-reported quantitative instruments. There are two different analysis tests based on the type of the constructs (reflective or formative). In the reflective construct the measures are all caused by a single underlying construct, while in formative model the measures all have an impact on (or cause) a single construct (Jarvis et al. 2003, p. 201). PRE is the only reflective construct in the proposed model and PSD and PR are the formative constructs.

\subsection{Reliability and Validity of The Constructs}

For the reflective construct of PRE, internal consistency reliability and indicator reliability are two reliability tests. The two validity tests are convergent validity and discriminant validity. As presented in Table 2, in both reliability tests the results of PRE construct could satisfy the conditions for reliability. Moreover, both validity tests were also successful in measuring the PRE construct according to Table 3.

Table 2. Reliability Assessment for Latent Construct of PRE

\begin{tabular}{clcc}
\hline Reliability Test & \multicolumn{1}{c}{ Measurement Method } & Criteria & Value \\
\hline \multirow{2}{*}{ Internal Consistency Reliability } & Cronbach's Alpha & $>.7$ &. $\mathbf{8 6 1}$ \\
& Composite Reliability & $>.7,<.95$ & $\mathbf{. 9 1 5}$ \\
& & & \\
Indicator Reliability & Outer Loading & $>.708$ & \\
& -Attention Restoration & & .892 \\
& -Clear Thoughts & &. $\mathbf{8 8 3}$ \\
& -Relaxation and Calmness & .877 \\
\hline
\end{tabular}


Table 3. Validity Assessment for Reflective Latent Construct of PRE

\begin{tabular}{llcccc}
\hline Validity Tests & Measurement Method & Criteria & PR & PSD & PRE \\
\hline $\begin{array}{l}\text { Convergent } \\
\text { Validity }\end{array}$ & AVE & $>.5$ & - & - &. $\mathbf{7 8 1}$ \\
& & & & & \\
Discriminant & a) Cross-Loadings & & & & \\
Validity & Attention Restoration & Highest & .557 & .451 & .892 \\
& $\quad$ Clearing Thoughts & Loading & .583 & .474 & .883 \\
& Relaxation \& Calmness & & .661 & .553 & .877 \\
& b) Fornell-Larcker Criterion & & & & \\
& PR & Highest & & & \\
& PSD & Loading & .655 & & \\
& PRE & & .684 & .562 &. $\mathbf{8 8 4}$ \\
\hline
\end{tabular}

Formative constructs of the proposed model are PSD and PR. In order to examine the quality of them, convergent validity, collinearity statistics among indicators and significance and relevance of outer weights of indicators have been conducted.

Convergent validity is done using a simple model connecting the formative construct to its single-item construct. For the path coefficient higher than 0.7 , the convergent validity of the construct is achieved. For PSD, the path coefficient value is 0.836 and for PR, it is 0.839 . Thus the convergent validity of formative constructs in this model is proven. Furthermore, the results of collinearity for proposed model are presented in Table 4 and all the indicators are within the required range.

Table 4. Collinearity Statistics with Outer VIF for Formative Latent Constructs of PSD and PR

\begin{tabular}{llcc}
\hline Construct & Indicator & Criteria & VIF \\
\hline & Culture & & $\mathbf{1 . 5 5 8}$ \\
& Nature & & $\mathbf{1 . 9 0 8}$ \\
PSD & Prospect & & $\mathbf{1 . 5 5 4}$ \\
& Refuge & $<5$ & $\mathbf{1 . 8 5 2}$ \\
& Rich in Species & & $\mathbf{1 . 8 9 6}$ \\
& Social & & $\mathbf{1 . 1 6 4}$ \\
& Space & & $\mathbf{1 . 3 7 8}$ \\
& Serene & & $\mathbf{1 . 3 6 8}$ \\
PR & & & \\
& Compatibility & & $\mathbf{1 . 6 3 5}$ \\
& Escape & & $\mathbf{1 . 2 8 6}$ \\
& Extent & $<5$ & $\mathbf{1 . 5 8 9}$ \\
& Fascination & & $\mathbf{1 . 6 9 9}$ \\
& Novelty & & $\mathbf{1 . 1 9 5}$ \\
\hline
\end{tabular}

Last step is to assess the significance of the construct measures in the model. Using Bootstrapping, if the p-value of an indicator is lower than 0.050 , then that indicator is significant. Otherwise, the outer loading of that indicator is checked if is equal or greater than 0.500 . If so, the indicator still is considered as significant. Otherwise, that indicator is insignificant in the model. According to Table 5 , all the variables for the constructs of PSD and PR except 'Culture' and 'Refuge' have p-value less than 0.050. 'Culture' has outer loading of $0.507 \geq 0.500$ and 'Refuge' has outer loading of $0.716 \geq 0.500$. Thus, both these variables are also considered as significant and is used in the assessment of the proposed model. 
Table 5. Indicator Significance Analysis for Formative Latent Constructs of PSD and PR

\begin{tabular}{|c|c|c|c|c|c|}
\hline Construct & Indicator & Std. Dev. & t-value & p-value & Outer Loading \\
\hline \multirow{8}{*}{ PSD } & Culture & .071 & 1.045 & .296 & .507 \\
\hline & Nature & .072 & 2.829 & .005 & $.729^{* *}$ \\
\hline & Prospect & .074 & 3.324 & .001 & $.709^{* *}$ \\
\hline & Refuge & .072 & 1.567 & .117 & .716 \\
\hline & Rich in Species & .073 & 3.095 & .002 & $.659^{* *}$ \\
\hline & Social & .057 & 2.784 & .005 & $.342^{* *}$ \\
\hline & Space & .070 & 3.740 & .000 & $.667^{* * *}$ \\
\hline & Serene & .065 & 5.762 & .000 & $.686^{* * *}$ \\
\hline \multirow{5}{*}{ PR } & Compatibility & .065 & 5.085 & .000 & $.791^{* * *}$ \\
\hline & Escape & .052 & 3.552 & .000 & $.579^{* * *}$ \\
\hline & Extent & .055 & 4.171 & .000 & $.738^{* * *}$ \\
\hline & Fascination & .059 & 7.803 & .000 & $.869^{* * *}$ \\
\hline & Novelty & .043 & 3.182 & .001 & $.485^{* *}$ \\
\hline
\end{tabular}

\subsection{Structural Model Assessment}

In a structural model the significant of relationship between the latent constructs is shown to support research theories and hypotheses. In evaluation of structural model, five analysis are taken place including: assessing path coefficients, evaluating the coefficients of determination, evaluating the effect size, assessing the predictive relevance.

The collinearity of the model is tested using predictor construct (PSD $\rightarrow$ PR, PR $\rightarrow$ PRE and PSD $\rightarrow$ PRE) tolerance and VIF. As shown in Table 6, all the paths' tolerance and VIF are between 0.20 and 5.00 and thus there is not collinearity problem in the model.

Table 6. Constructs' Collinearity Examinations using VIF

\begin{tabular}{lccc}
\hline Link & Criteria & Tolerance & VIF \\
\hline PSD $\rightarrow$ PRE & Tolerance $>.20$ &. $\mathbf{5 7 1}$ & $\mathbf{1 . 7 5 1}$ \\
$\mathrm{PSD} \rightarrow \mathrm{PR}$ & and & $\mathbf{1 . 0 0 0}$ & $\mathbf{1 . 0 0 0}$ \\
$\mathrm{PR} \rightarrow \mathrm{PRE}$ & $\mathrm{VIF}<5$ & $\mathbf{. 5 7 1}$ & $\mathbf{1 . 7 5 1}$ \\
\hline
\end{tabular}

In evaluation of structural model, coefficient of determination $\left(R^{2}\right.$ value) shows how much of endogenous construct (dependent variable) is predicted by exogenous constructs (independent variable) connected to it. Value less than .25 shows weak, from 0.25 to 0.50 shows moderate, from 0.50 to 0.75 shows substantial and bigger than that shows high level of predictive accuracy. As presented in Table. 7, a moderate prediction accuracy of the latent constructs exists in the model.

Table 7. Constructs $R^{2}$ Results

\begin{tabular}{lcccc}
\hline Construct & $\mathbf{R}^{\mathbf{2}}$ & Std. Dev. & t-value & p-value \\
\hline PR & $.429^{* * *}$ & .034 & 12.555 & .000 \\
\hline PRE &. .900 $^{* * *}$ & .034 & 14.249 & .000 \\
\hline - Significance levels: ${ }^{*} p<.05,{ }^{* *} p<.01,{ }^{* * *} p<.001$.
\end{tabular}

The effect size $f^{2}$ : The effect size $f^{2}$ demonstrates that how much predictor constructs of PSD and PR (independent variables) are contributing to the coefficient of determination ( $R^{2}$ value) of endogenous latent variables (PR and PRE). Values of 0.02, 0.15, 0.35 and greater are interpreted as small, medium and large effect size. As shown in Table 8 there is large effect of PSD on PR, medium effect of PR on PRE and small effect of PSD on PRE. 
Table 8. The Effect Size of The Paths in The Model

\begin{tabular}{lc}
\hline Link & $\mathbf{f}^{2}$ \\
\hline PSD $\rightarrow$ PRE & 0.044 \\
PSD $\rightarrow$ PR & 0.751 \\
$P R \rightarrow$ PRE & 0.342 \\
\hline
\end{tabular}

Blindfolding and predictive relevance $Q^{2}$ : Measurement of $Q^{2}$ displays the model's predictive relevance. Table 9 and 10 are blindfolding measurements for both indicators and endogenous constructs (PR and PRE), where $Q^{2}>0.000$ means that the construct or indicator has predictive relevance. In both tables, SSO indicates the Sum of the Squared Observations and SSE is the Sum of Squared Prediction Errors. Values of $0.02,0.15$, and 0.35 of $Q^{2}$ show small, medium and large predictive relevance of an endogenous construct. For the proposed model, there is medium predictability for PR by PSD and large predictability for PRE by PSD and PR.

Table 9. Blindfolding to Examine Cross-Validated Redundancy for Endogenous Constructs

\begin{tabular}{lccc}
\hline Construct & SSO & SSE & Q $^{2}$ \\
\hline PSD & $3,552.00$ & $3,552.000$ & \\
PR & $2,220.00$ & $1,782.639$ & .197 \\
PRE & $1,332.00$ & 857.553 & .356 \\
\hline
\end{tabular}

Table 10. Blindfolding to Examine Cross-Validated Redundancy for Indicators

\begin{tabular}{llccc}
\hline Construct & Indicator & SSO & SSE & $\mathbf{Q}^{2}$ \\
\hline \multirow{4}{*}{ PSD } & Culture & 444 & 444 & \\
& Nature & 444 & 444 & \\
& Prospect & 444 & 444 & \\
& Refuge & 444 & 444 & \\
& Rich in Species & 444 & 444 & \\
& Social & 444 & 444 & \\
& Space & 444 & 444 & \\
& Serene & 444 & 444 & \\
& & & & \\
& Compatibility & 444 & 346.347 & .220 \\
& Escape & 444 & 397.704 & .104 \\
& Extent & 444 & 341.866 & .230 \\
& Fascination & 444 & 297.359 & .330 \\
& Novelty & 444 & 399.363 & .101 \\
& Attention Restoration & 444 & 305.497 & .312 \\
& Clearing Thoughts & 444 & 294.112 & .338 \\
& Relaxation \& Calmness & 444 & 257.944 & .419 \\
\hline
\end{tabular}

Significance and Relevance: The significances of the path coefficients are examined using Bootstrapping method. As shown in Table 11, all the p-values for paths are lower than 0.05. Moreover, $\mathrm{T}$ Statistics (t-value) for all paths are above 1.96. Thus, all the paths are significant in this model. 
Table 11. Examining the Significance of Path Coefficients Using Bootstrapping

\begin{tabular}{|c|c|c|c|c|c|c|}
\hline \multirow[t]{2}{*}{ Link } & \multirow{2}{*}{ Path Coefficient } & \multirow{2}{*}{ Std. Dev. } & \multicolumn{2}{|c|}{ t-value } & \multicolumn{2}{|c|}{ p-values } \\
\hline & & & Criteria & Value & Criteria & Value \\
\hline $\mathrm{PSD} \rightarrow \mathrm{PRE}$ & $.199^{* * *}$ & .046 & & 4.310 & & .000 \\
\hline $\mathrm{PSD} \rightarrow \mathrm{PR}$ & $.655^{* * *}$ & .026 & $>1.96$ & 25.276 & $<.05$ & .000 \\
\hline $\mathrm{PR} \rightarrow \mathrm{PRE}$ & $.553^{* * *}$ & .041 & & 13.423 & & .000 \\
\hline
\end{tabular}

\subsection{Model Analysis Results}

In model analysis, the first relation to investigate is the link between PSD and PRE. Although the path coefficient of PSD $\rightarrow$ PRE is as low as 0.199, the mediation effect of PR also should be taken into account. Thus, the total effect of this link is calculated and presented in Table 12. As shown in this Table, the total effect of PSD on PRE is as high as 0.562. Therefore, the existence of the link between PSD and PRE is supported. In addition to PSD $\rightarrow$ PRE, the other two links of this model (PSD $\rightarrow$ PR and PR $\rightarrow$ PRE) show a high total effect with values 0.655 and 0.553 , respectively. Thus, the existence of the strong links among all constructs of this model has been supported.

Table 12. Path Coefficient Results

\begin{tabular}{lccc}
\hline Link & Path Coefficients & Indirect Effect & Total Effect \\
\hline PSD $\rightarrow$ PRE & .199 & .362 & .562 \\
PSD $\rightarrow$ PR & .655 & & .655 \\
PR $\rightarrow$ PRE & .553 & & .553 \\
\hline
\end{tabular}

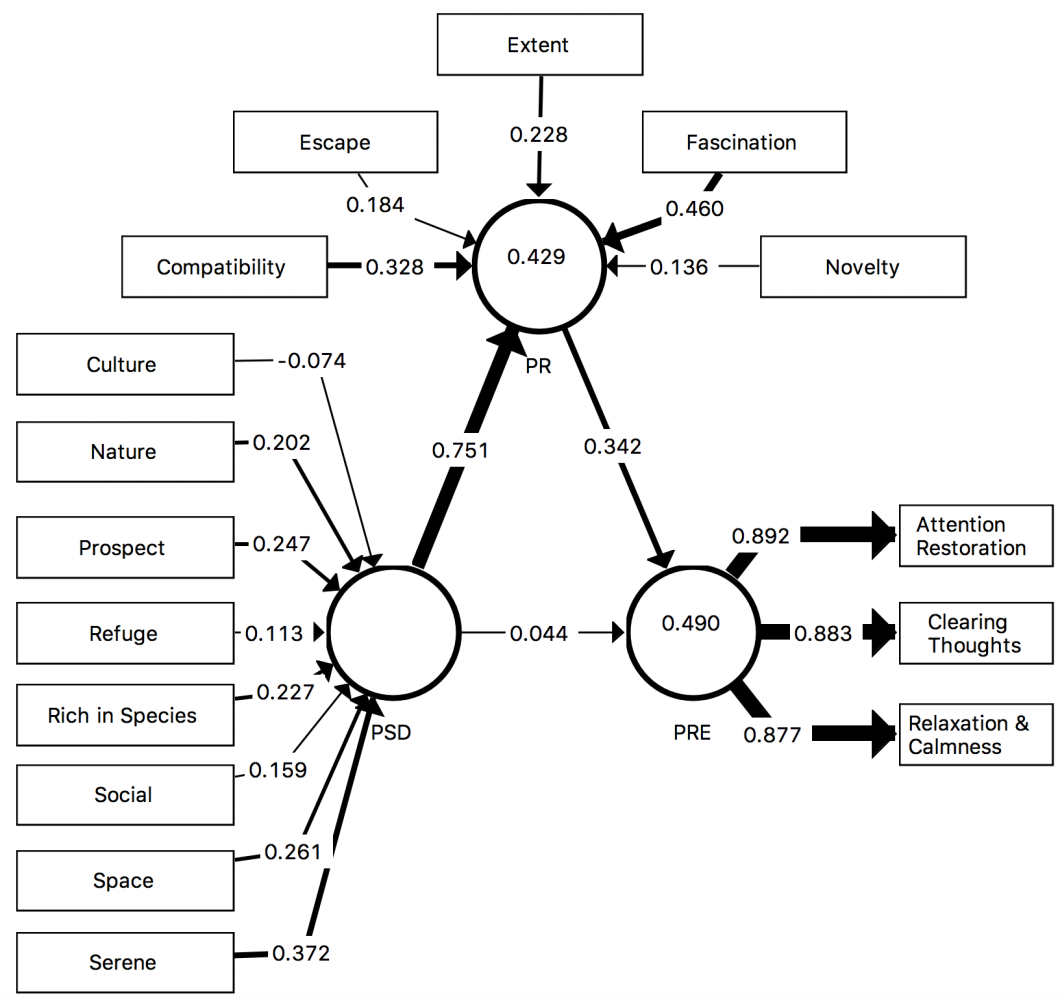

Figure 2. Result of PLS Analysis on Proposed Model 
Figure 2 demonstrates a complete view to the links and relations of variables and constructs of the model. The highlighted paths have higher effect in the model compared to other ones. For instance, $\mathrm{PSD} \rightarrow \mathrm{PR}$ is highlighted but not PSD $\rightarrow$ PRE comparing the two path coefficients of 0.655 and 0.199 .

In this part, the effect of measurement variables to develop their construct is examined. The only reflective construct in the model is PRE with three measurement variables of 'Attention Restoration','Clearing Thoughts', 'Relaxation \& Calmness'. As shown in Table 13, all three measurement variables of construct PRE are significant $(\mathrm{p}$-value $=0.000)$ with high $\mathrm{t}$-values. Moreover, all three variables have almost the same outer loading values which demonstrates the importance of presence of all of them in developing the construct of PRE.

Table 13. Outer Loading of Reflective Construct PRE

\begin{tabular}{lcccc}
\hline Indicator & Outer Loading & Std. Dev. & t-value & p-values \\
\hline Attention Restoration & $.892^{* * *}$ & .012 & 76.811 & .000 \\
Clearing Thoughts &. $\mathbf{8 8 3}^{* * *}$ & .013 & 67.018 & .000 \\
Relaxation \& Calmness &. $\mathbf{8 7 7}^{* * *}$ & .011 & 80.151 & .000 \\
\hline - Significance levels: ${ }^{*} p<.05,{ }^{* *} p<.01{ }^{* * *} p<.001$. & &
\end{tabular}

There are two formative constructs in the model PSD and PR. The result of outer weights for measurement variables of these two constructs is presented in Table 14. PSD consists of 8 measurement variables. As shown, 'Culture' and 'Refuge' have not significant effect in PSD construct with $p$-values of 0.287 and 0.121 respectively. From the rest of measurement variables 'Serene' has the highest outer weight (0.372). 'Space', 'Prospect', 'Rich in Species' and 'Nature' are the next important measurement variables in PSD construct. The lowest and yet significant measurement variable in PSD construct is 'Social' with outer weight of 0.159 .

The second formative construct in the model is PR. PR consists of 5 measurement variables and the result of outer weight analysis of them is presented in Table 14. All these variable are significant with $p$ $<0.050$. Among these variables, 'Fascination' has the highest outer weight followed by 'Compatibility'. Other three measurement variables 'Extent', 'Escape' and 'Novelty' have relatively low outer weight compared to two aforementioned.

Table 14. Outer Weights of Formative Constructs of PSD and PR

\begin{tabular}{|c|c|c|c|c|c|}
\hline Construct & Indicator & Outer Weight & Std. Dev. & t-value & p-values \\
\hline \multirow{8}{*}{ PSD } & Culture & -.074 & .070 & 1.064 & .287 \\
\hline & Nature & $.202^{* *}$ & .072 & 2.821 & .005 \\
\hline & Prospect & $.247^{* *}$ & .074 & 3.347 & .001 \\
\hline & Refuge & .113 & .073 & 1.551 & .121 \\
\hline & Rich in Species & $.227^{* *}$ & .074 & 3.057 & .002 \\
\hline & Social & $.159^{* *}$ & .056 & 2.833 & .005 \\
\hline & Space & $.261^{* * *}$ & .070 & 3.739 & .000 \\
\hline & Serene & $.372^{* * *}$ & .065 & 5.687 & .000 \\
\hline \multirow{5}{*}{ PR } & Compatibility & $.328^{* * *}$ & .065 & 5.076 & .000 \\
\hline & Escape & $.184^{* * *}$ & .052 & 3.534 & .000 \\
\hline & Extent & $.228^{* * *}$ & .055 & 4.131 & .000 \\
\hline & Fascination & $.460^{* * *}$ & .059 & 7.818 & .000 \\
\hline & Novelty & $.136^{* *}$ & .043 & 3.146 & .002 \\
\hline
\end{tabular}

- Significance levels: ${ }^{*} p<.05,{ }^{* *} p<.01,{ }^{* * *} p<.001$.

\subsection{Mediator Effect Analysis of PR}

To show that the PR has a mediation effect in the model, following steps are done. Firstly, the path coefficient of PSD $\rightarrow$ PRE is measured in the absence of construct PR. The PLS and Bootstrapping 
results of it are as shown in Table 15. As shown, there is a significant relation between PSD and PRE (T Statistic $17.628>1.96$ and p-value $0.00<0.05$ ) with the path coefficient of 0.569 . Secondly, for indirect analysis, PR is included as a mediator for the relation between PSD and PRE where the proposed model is shown in Fig 2. The PLS and Bootstrapping results (Table 11) show that PR is a partial mediator for PSD to PRE relation. The first reason is that both the paths of PSD to PR and PR to PRE are significant ( $t$-value $13.459>1.96 \& 25.428>1.96$ ) where $\mathrm{p}$-values are less than 0.05 . Next, the significant of direct relation between PSD to PRE decreased from 17.628 to 4.365, which demonstrates the presence of the mediator effect in the model. PR acts as a partial moderator in the model as the path between PSD and PRE in the mediator model is still significant. To examine this, the Variance Accounted For (VAF) is calculated in Eq. 2. Based on (Hair et al. 2016), The VAF bigger than 0.8 means full mediation and a value between 0.2 to 0.8 is partial mediation effect. The result of VAF in this model with mediator effect is 0.645 . Therefore, PR has a partial mediation rule and indirectly, the link between PSD and PRE is effected via the mediate effect of this construct.

Table 15. PLS and Bootstrapping result for relation between PSD and PRE without mediation effect

\begin{tabular}{lcccc}
\hline Link & Path Coefficient & Std. Dev. & t-value & p-values \\
\hline PSD $\rightarrow$ PRE & $.569^{* * *}$ & .032 & 17.628 & .000 \\
\hline - Significance levels: ${ }^{*} p<.05,{ }^{* *} p<.01,{ }^{* * *} p<.001$. &
\end{tabular}

$$
\begin{array}{r}
V A F=\left(p_{12} * p_{23}\right) /\left(p_{12} * p_{23}+p_{13}\right) \\
V A F=(.655 * .553) /(.655 * .553+.199) \\
V A F=.645
\end{array}
$$

\section{Discussion}

Studies that have investigated the impact of PSD on perceived restorativeness and restoration experience are few. The purpose of this study was to examine the restoration experience through impacts of PSDs and perceived restorativeness characteristics, in order to provide recommendations for designing health-promoting environments. Also, it examined whether perceived restorativeness would mediate the effect of PSD on restoration experience as well as what are the influential PSDs and restorativeness characteristics in this process.

The result on PSD $\rightarrow$ PRE show positive significant relationship (support H1). This finding is accordance with previous studies that showed perceived green space related qualities could be associated with better mental health, well-being and stress restoration (Annerstedt et al. 2012; De Jong et al. 2011; Memari et al. 2017). The finding is also correspond with the study on university campus environments that showed perceived greenness is positively related with higher level of students' quality of life (Hipp et al. 2016).

The result on PSD $\rightarrow$ PR supports the second hypothesis (H2) that perceived open space qualities have influence on evaluation of restorativeness characteristics. In a more similar attempt, through expert objective measurement approach, it showed that PSDs can positively be associated with users judgement of restorativeness value of urban open green spaces (Peschardt and Stigsdotter 2013). However, in this study, this relationship is established through subjective experience of PSDs and potential restorativeness characteristics, rather than experts objective evaluation approach, which may cause different views in evaluation of qualities in landscape perception (Qiu and Nielsen 2015).

The result on PR $\rightarrow$ PRE supports the third hypothesis (H3) that perceived restorativeness has significant effect on restoration experience. It is similar with the findings of Felsten (2009), who demonstrated exposure to restorativeness characteristics of simulated nature in indoor campus settings has recovery effects. This study found that experience of restorativeness in campus outdoor open 
spaces has positive effect on students psychological restoration. Based on ART, the essential condition for experience of restorative outcomes is when environment provides restorativeness values (Marselle et al. 2016). Campus open space permits restorativeness experience and is capable of promoting experience of psychological restoration.

The direct, indirect and total effect analysis as well as VAF calculation supports that PR provides a medium mediation effect to explain the impact of PSD on PRE (H4). This is congruence with earlier studies that showed mediation effect of perceived restorativeness on the effect of environmental qualities on emotional well-being (Carrus et al. 2015; Marselle et al. 2016) and university students better quality of life Hipp et al. (2016). The association of PSD and restorativeness has been demonstrated by previous studies (Peschardt and Stigsdotter 2013; Stigsdotter et al. 2017; Stoltz et al. 2016). To our knowledge, this is the first study in which the mediator effect of perceived restorativeness on the relationship between PSD and restoration experience is examined. Similar with other studies that showed mediation effect of perceived restorativeness (Carrus et al. 2015; Hipp et al. 2016; Marselle et al. 2016), this study showed that perceived restorativeness is important in shaping relationship between PSD and restoration experience. Greater restoration experience highly depends on effect of PSDs on perceived restorativeness, and then experience of restorative outcomes.

All five measurement variables of PR were found with positive significant effects (Q1). However, the highest effect is occurred by 'fascination' and 'compatibility'. Campus open spaces, which are rich in providing of soft fascination experiences and supporting students required activities or behaviors highly lead to restoration experience. Similar finding with less experience of extent has been addressed in Felsten (2009), where campus indoor settings with no views of nature did not induce great mental restoration. Campus outdoor open spaces, however, is suggested to be ideal setting in experience of restoration by engaging to abundance restorative objects (Lau et al. 2014; Seitz et al. 2014). So, it is expected that such settings allow a deep immersion, which facilitate a sense of distance from sustained academic life strains. It could be because students typically use campus open spaces for their daily activities and in such settings they still continue stressful feelings which are associated with their campus life. That might be the reason why they are not became fully immersed in such settings to perceive more psychologically and physically aspects of being away from daily responsibilities. Support to this explanation is, when students were asked to visit a natural place that they believe to be beneficial for their mental health development, they visited a separate natural place from the context of their everyday campus to relief from daily concerns (Windhorst and Williams 2015). Recreational visits on campus open spaces would not give students shift attention away from 'hard fascination' experiences that associated with campus structure such as lectures, busy course schedules, assignments, exams and so on (Seitz et al. 2014).

A number of measurement variables of PSD was found with significant effects in the model (Q2). The strongest effect is by perceiving the 'serene' quality followed by perceived qualities of 'space', 'prospect', 'rich in species' and 'nature'. 'Serene' was the most cited quality in preference for urban green open spaces (Grahn and Stigsdotter 2010), experience of restorativeness (Peschardt and Stigsdotter 2013; Stigsdotter et al. 2017) and stress restoration (Memari et al. 2017). Sense of 'space' was found with effect on evaluation of perceived restorativeness (Peschardt and Stigsdotter 2013) and decrease in poor mental health (Annerstedt et al. 2012). A setting with broad vista view ('prospect') has restorativeness potential as required less direct attention Gatersleben and Andrews (2013) and is effective during a stress recovery stage Wang et al. (2016). People inherently love living things in nature and was shown that environments with 'rich in species' have positive effect on their emotional restoration (Carrus et al. 2015; Marselle et al. 2016) and mental well- being Wolf et al. (2017). Strong feelings for component of 'nature' reflects its biological effect, which found with positive outcomes (Grahn and Stigsdotter 2010). Therefore, it is logically plausible that campus open spaces, in which there are sense of silent, peaceful and undisturbed environment ('serene'), lots of sunny and shady areas without signals that demand attention ('space'), landscapes with open view areas 'prospect', diverse expressions of life in nature 
'rich in species' and sensation of naturally growing greenery 'nature' are associated with perceived restorativeness and emerged feelings of restoration.

The finding in less association of 'social' is similar with studies with focus on nature- assisted therapy program (Grahn et al. 2010). A possible explanation might be that students who encountered with sever stress and have socialization in class programs expect to find campus open spaces as places of tranquillity and solitude. This dimension may be a desired quality of open spaces for people who are living in cities (Peschardt and Stigsdotter 2013). The result of separation and solitude by Windhorst and Williams (2015) demonstrated that for experience of better mental health, university students preferred to visit separate natural places from the context of campus to remove themselves from social environment of campus life.

Perceived restorativeness and restoration experience are associated neither with perceived quality of 'refuge' and nor with 'culture'. Green open spaces with cultural qualities are interesting places for experience of well-being outcomes (De Jong et al. 2012) and perceived restorativeness (Peschardt and Stigsdotter 2013). In human nature interaction, it explained that people first sought simple objects and experience of 'culture' found to be important only when people began to explore the environments for symbolic values Grahn et al. (2010); Stigsdotter et al. (2017). Although, the study by Windhorst and Williams (2015) highlighted the importance of cultural features in visitation to natural places for university students mental health development, the effect was not found in open spaces inside campus. The restorative potential of 'refuge' quality is that this dimension offer sitting in privacy (Peschardt and Stigsdotter 2013; Stigsdotter et al. 2017). However, it seems that university students with more experiences of indoor campus settings prefer campus open spaces with visibility of views than landscapes with enclosed areas.

\section{Limitations and Recommendations}

There are some limitations in this study, which should be considered in the future studies. The non-experimental approach in measurement of PSDs, PR and PRE might be the limitation of this study. The first reason is that it could not monitor restorative changes in the subjects' psychological, physiological and emotional states. Second, it could not monitor environmental changes that reflect on perception of PSDs. Further research is needed to elucidate them by an experimental approach, to obtain data from the same respondents repeatedly over time and by motoring changes in perceiving of PSDs, in order to gain knowledge on the effect of PSDs over the changing seasons in development of better psycho-physiological and emotional conditions. A comparative study on evaluation of PSDs would be valuable to find the differences and similarities of experts as open space designers and lay-public as its users. Little knowledge of designers and non-designers' perception of these characteristics has been addressed, yet. Although restoration experience is supported by the proposed characteristics, further research should be accounted for other missing mediating variables to find more possible effects on other aspects such as students quality of life or achievement of academic performance.

\section{Implications of Findings}

This study provides an understanding of the factors that influenced perceived restoration experience. The findings can be used within landscape architecture and health development to design restorative supportive environments. To do this, landscape architects and health planners should design places with a combination of restorativeness characteristics and salutary qualities which support psychological restoration and consequently affect the whole mental health. The restorativeness characteristics and supportive salutary qualities of open spaces is not limited by geographical boundaries. Thus, it can be applied in other cases or at least environments that sharing the same population with this study. The result are expected to be the same in other educational settings in Malaysia. 


\section{Conclusion}

As the contribution of environment qualities and perceived restorativeness for positive outcomes is required, understanding the impact of PSD and perceived restorativeness on perceived restoration experience is important. The findings on structural model supports significant relationship between constructs of PSD, PR and PRE. Moreover, the model explains half of the perceived restoration of the university students in campus open spaces, through the effect of PSD and PR. In this relationships, the greatest effect was predicted through the mediating effect of perceived restorativeness. The influential PSD variables were 'serene', 'space', 'prospect', 'rich in species' and 'nature' and PR variables were 'fascination' and 'compatibility'. Integration of these PSD and PR qualities provided restorative outcomes of 'attention restoration', 'clearing thoughts' and 'relaxation $\mathcal{E}$ calmness' almost equally.

Author Contributions: This research has been done by Fahimeh Malekinezhad under supervision of Prof. Dr. Hasanuddin bin Lamit

Conflicts of Interest: The authors declare no conflict of interest.

\section{References}

Annerstedt, M., P. O. Östergren, J. Björk, P. Grahn, E. Skärbäck, and P. \& Währborg. 2012. Green Qualities in the Neighbourhood and Mental Health - Results from a Longitudinal Cohort Study in Southern Sweden. BMC Public Health 12(1), 337.

Asztalos, M., K. Wijndaele, I. De Bourdeaudhuij, R. Philippaerts, L. Matton, N. Duvigneaud, M. Thomis, W. Duquet, J. Lefevre, and Cardon, G.. 2009. Specific Associations between Types of Physical Activity and Components of Mental Health. Journal of Science and Medicine in Sport 12, 468-474.

Baghurst, T. and B. C. Kelley. 2014. An Examination of Stress in College Students Over the Course of a Semester. Health Promotion Practice 15(3), 438-447.

Bagot, K. L.. 2004. Perceived Restorative Components: A Scale for Children. Children, Youth and Environments 14(1), 107-129.

Bengtsson, A. and P. Grahn. 2014. Outdoor Environments in Healthcare Settings: A Quality Evaluation Tool for Use in Designing Healthcare Gardens. Urban Forestry \& Urban Greening 13(4), 878-891.

Bratman, G. N., G. C. Daily, B. J. Levy, and J. J. \& Gross. 2015. The Benefits of Nature Experience: Improved Affect and Cognition. Landscape and Urban Planning 138, 41-50.

Carrus, G., M. Scopelliti, R. Lafortezza, G. Colangelo, F. Ferrini, . Salbitano, F., and G. \& Sanesi. 2015. Go Greener, Feel Better? The Positive Effects of Biodiversity on the Well-being of Individuals Visiting Urban and Peri-urban Green Areas. Landscape and Urban Planning 134, 221-228.

De Jong, K., M. Albin, E. Skärbäck, P. Grahn, and J. Björk. 2012. Perceived Green Qualities were Associated with Neighborhood Satisfaction, Physical Activity, and General Health: Results from a Cross-Sectional Study in Suburban and Rural Scania, Southern Sweden. Health and Place 18, 1374-1380.

De Jong, K., M. Albin, E. Skärbäck, P. Grahn, J. Wadbro, J. Merlo, and J. Björk. 2011. Area-aggregated Assessments of Perceived Environmental Attributes May Overcome Single-source Bias in Studies of Green Environments and Health: Results from a Cross-sectional Survey in Southern Sweden. Environmental Health 10(1), 1-11.

De Vaus, D.. 2002. Surveys in Social Research. Allen and Unwin.

Felsten, G.. 2009. Where to Take a Study Break on the College Campus: An Attention Restoration Theory Perspective. Journal of Environmental Psychology 29(1), 160-167.

Gatersleben, B. and M. Andrews. 2013. When Walking in Nature is Not Restorative-The Role of Prospect and Refuge. Health \& Place 20, 91-101.

Grahn, P.. 1991. Landscapes in Our Minds: People's Choice of Recreative Places in Towns. Landscape Research 16(1), 11-19.

Grahn, P., U. A. Stigsdotter, and A. M. Berggren-Barring. 2005. A Planning Tool for Designing Sustainable and Healthy Cities. The Importance of Experienced Characteristics in Urban Green Open Spaces for People's Health and Well-Being. In Quality and Significance of Green Urban Areas, pp. 29-38. Van Hall Larwnstein University of Professional Education, Netherlands. 
Grahn, P. and U. K. Stigsdotter. 2010. The Relation between Perceived Sensory Dimensions of Urban Green Space and Stress Restoration. Landscape and Urban Planning 94(3), 264-275.

Grahn, P., C. Tenngart, I. Ulrika, and I. Bengtsson. 2010. Using Affordances as a Health-promoting Tool in a Therapeutic Garden. In Innovative Approaches to Researching Landscape and Health, pp. 116-154.

Hair, J. F., G. Hult, C. Ringle, and M. Sarstedt. 2016. A Primer on Partial Least Squares Structural Equation Modeling (PLS-SEM). Sage Publications.

Haluza, D., S. Simic, J. Höltge, R. Cervinka, and H. Moshammer. 2014. Connectedness to Nature and Public (Skin) Health Perspectives: Results of a Representative, Population-Based Survey among Austrian Residents. International Journal of Environmental Research and Public Health 11(1), 1176-1191.

Han, K. T.. 2003. A Reliable and Valid Self-rating Measure of the Restorative Quality of Natural Environments. Landscape and Urban Planning 64(4), 209-232.

Hansmann, R., S. M. Hug, and K. \& Seeland. 2007. Restoration and Stress Relief through Physical Activities in Forests and Parks. Urban Forestry \& Urban Greening 6(4), 213-225.

Hipp, J. A., G. B. Gulwadi, S. Alves, and S. Sequeira. 2016. The Relationship between Perceived Greenness and Perceived Restorativeness of University Campuses and Student-Reported Quality of Life. Environment and Behavior 48(10), 1292-1308.

Ivarsson, C. T. and C. M. \& Hagerhall. 2008. The Perceived Restorativeness of Gardens - Assessing the Restorativeness of a Mixed Built and Natural Scene Type. Urban Forestry E Urban Greening 7(2), 107-118.

Jarvis, C., S. Mackenzie, P. Podsakoff, D. Mick, and W. Bearden. 2003. A Critical Review of Construct Indicators and Measurement Model Misspecification in Marketing and Consumer Research. Journal of Consumer Research 30(2), 199-218.

Kaplan, S.. 1995. The Restorative Benefits of Nature: Toward an Integrative Framework. Journal of Environmental Psychology 15(3), 169-182.

Korpela, K. M., M. Ylén, L. Tyrväinen, and H. Silvennoinen. 2008. Determinants of Restorative Experiences in Everyday Favorite Places. Health \& Place 14(4), 636-652.

Kumar, R.. 2011. Research Methodology: A Step-by-Step Guide for Beginners. SAGE.

Lau, S. S. Y., Z. Gou, and Y. Liu. 2014. Healthy Campus by Open Space Design: Approaches and Guidelines. Frontiers of Architectural Research 3(4), 452-467.

Lehto, X. Y.. 2013. Assessing the Perceived Restorative Qualities of Vacation Destinations. Journal of Travel Research 52(3), 325-339.

Marselle, M., K. Irvine, A. Lorenzo-Arribas, and S. Warber. 2015. Moving beyond Green: Exploring the Relationship of Environment Type and Indicators of Perceived Environmental Quality on Emotional Well-Being following Group Walks. International Journal of Environmental Research and Public Health 12(1), 106-130.

Marselle, M. R., K. N. Irvine, A. Lorenzo-Arribas, and S. L. Warber. 2016. Does Perceived Restorativeness Mediate the Effects of Perceived Biodiversity and Perceived Naturalness on Emotional Well-Being Following Group Walks in Nature? Journal of Environmental Psychology 46, 217-232.

Memari, S., M. Pazhouhanfar, and A. Nourtaghani. 2017. Relationship between Perceived Sensory Dimensions and Stress Restoration in Care Settings. Urban Forestry and Urban Greening 26(May), 104-113.

Nordh, H., T. Hartig, C. Hagerhall, and G. Fry. 2009. Components of Small Urban Parks that Predict the Possibility for Restoration. Urban Forestry \& Urban Greening 8(4), 225-235.

Pals, R., L. Steg, F. Siero, and K. van der Zee. 2009. Development of the PRCQ: A Measure of Perceived Restorative Characteristics of Zoo Attractions. Journal of Environmental Psychology 29(4), 441-449.

Pasini, M., R. Berto, M. Brondino, R. Hall, and C. Ortner. 2014. How to Measure the Restorative Quality of Environments: The PRS-11. Procedia - Social and Behavioral Sciences 159, $293-297$.

Peschardt, K. K. and U. K. Stigsdotter. 2013. Associations between Park Characteristics and Perceived Restorativeness of Small Public Urban Green Spaces. Landscape and Urban Planning 112(1), $26-39$.

Qiu, L. and A. B. Nielsen. 2015. Are Perceived Sensory Dimensions a Reliable Tool for Urban Green Space Assessment and Planning? Landscape Research 40(7), 834-854.

Ringle, C. M., S. Wende, and J. M. Becker. 2015. SmartPLS 3. Boenningstedt: SmartPLS GmbH, http://www. smartpls. com.

Scopelliti, M., G. Carrus, F. Cini, S. Mastandrea, F. Ferrini, R. Lafortezza, M. Agrimi, F. Salbitano, Sanesi, G., and P. Semenzato. 2012. Biodiversity, Perceived Restorativeness, and Benefits of Nature. Vulnerability, Risks, and Complexity: Impacts of Global Change on Human Habitats 3, 255-269. 
Scopelliti, M. and M. Vittoria Giuliani. 2004. Choosing Restorative Environments Across the Lifespan: A Matter of Place Eexperience. Journal of Environmental Psychology 24(4), 423-437.

Seitz, C. M., R. F. Reese, R. W. Strack, S. Frantz, and B. West. 2014. Identifying and Improving Green Spaces on a College Campus: A Photovoice Study. Ecopsychology 6(2), 98-108.

Skarback, E. and P. Grahn. 2016. Presence of Cars in Working Environments Impairs Perception of Sensory Dimensions. In INTER-NOISE and NOISE-CON Congress and Conference Proceedings, Number 1, pp. $943-949$.

Skärbäck, E., L. Wen, S. Aleksandrova, and P. Grahn. 2015. The Serene and Other Affordances in Demanding Contexts. IFLA 52nd World Congress, 1-9.

Stigsdotter, U. K., S. S. Corazon, U. Sidenius, A. D. Refshauge, and P. Grahn. 2017. Forest Mental Health Promotion-Using Perceived Sensory Dimensions to Elicit Restorative Responses. Landscape and Urban Planning 160, 1-15.

Stoltz, J., Y. Lundell, E. Skärbäck, M. A. van den Bosch, P. Grahn, E. M. Nordström, and A. Dolling. 2016. Planning for Restorative Forests: Describing Stress-reducing Qualities of Forest Stands Using Available Forest Stand Data. European Journal of Forest Research 135(5), 803-813.

Wang, X., S. Rodiek, C. Wu, Y. Chen, and Y. Li. 2016. Stress Recovery and Restorative Effects of Viewing Different Urban Park Senes in Shanghai, China. Urban Forestry and Urban Greening 15, 112-122.

White, M. P., S. Pahl, K. Ashbullby, S. Herbert, and M. H. Depledge. 2013. Feelings of Restoration from Recent Nature Visits. Journal of Environmental Psychology 35, 40-51.

Windhorst, E. and A. Williams. 2015. "It's Like a Different World": Natural Places, Post-secondary Students, and Mental Health. Health \& Place 34, 241-250.

Wolf, L. J., S. zu Ermgassen, A. Balmford, M. White, and N. Weinstein. 2017. Is Variety the Spice of Life? An Experimental Investigation into the Effects of Species Richness on Self-Reported Mental Well-Being. PloS one 12(1), e0170225. 